\title{
AN OSCILLATORY NEURAL NETWORK SCHEME FOR TEMPORAL ENCODING AND STIMULUS RECOGNITION
}

\author{
T. Ward, Student Member IEEE, A. de Paor, \\ Dept. of Electronic and Electrical Engineering, National University of Ireland, Dublin, Dublin 4, \\ Ireland.
}

\begin{abstract}
A novel computational neuro-architecture based on the phase resetting properties of physiologically based neural oscillators is proposed. Analog input variables are encoded in the patterns of the firing times with individual recognition units operating as radial basis-functions. Keywords- Neural Networks, Hodgkin-Huxley.
\end{abstract}

\section{INTRODUCTION}

Temporal neural networks (TNN) hold great promise for research in computational neuroscience as the rate coding hypothesis is not sufficient to account for all the extraordinary computational speeds encountered in neural processing. However, little is known regarding how these temporal codes are generated. A tentative solution is proposed based on the phase response surfaces of modelled neural oscillators. The outputs of such encoding 'neurons' are volleys of neural pulses with relative phases corresponding to the input pattern. Recognition is achieved using 'coincidence detector' cells as proposed by Hopfield [1]. We use a bank of individual synchronised oscillatory reduced-order HodgkinHuxley (ORHH) neurons[2] simulated in software feeding into a single cell through a network of delays. Each output neuron corresponds to a "stored" pattern and uses an activation function based on the summed excitatory post synaptic potentials (EPSPs).

\section{METHODS}

The co-phase [3] surface $Z(\theta, D)$ for the $O R H H$ neuron is found experimentally using stimuli of varying amplitudes over the input phases $\theta=[0,1]$. This surface can for certain $\theta$ be reduced to a monotonic function $g(I)$ (e.g. Fig. 1) which assigns a unique output delay $\tau$ in the timing of the next action potential, for a current pulse of amplitude $I$.

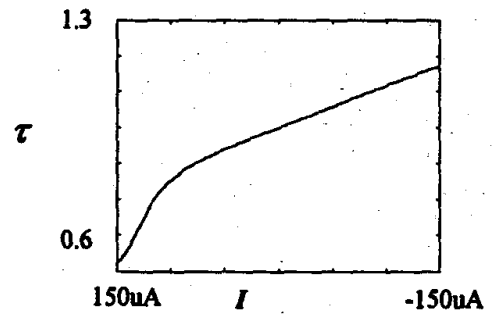

Figure 1 Section through the co-phase surface for a fixed $Q$ The problem is that given a set of $\boldsymbol{K}$ stimuli $a, b, c$, etc. to which the system is tuned, characterised by a set of numbers

$$
Z^{u}=\left\{Z_{1}^{u}, Z_{2}^{u}, \ldots, Z_{N}^{u}\right\}
$$

then for a novel stimulus $u$ the system must respond most strongly with the output neuron corresponding to the "closest" stored pattern. The novel stimulus $u$ is transformed by some pre-processing neural system $G p$ to yield a set of current pulses of uniform duration $T^{3}$ with amplitudes $I_{F}=G p(Z)$. which in turn feed into a bank of synchronised uniform $O R H H$ neurons at a specific phase $\theta$ which causes a phase shift $F g(I)$ such that

$$
Z^{u}=\left\{Z_{1}^{u}, Z_{2}^{u}, \ldots, Z_{N}^{u}\right\} \stackrel{G p}{\longrightarrow} I_{T^{u}}^{u}=\left\{I_{1}^{u}, I_{2}^{u}, \ldots, I_{N}^{u}\right\}
$$

and

$$
I_{T^{*}}^{u}=\left\{I_{1}^{u}, I_{2}^{u}, \ldots, I_{N}^{u}\right\} \stackrel{g}{\longrightarrow}\left\{\tau_{1}^{u}, \tau_{2}^{u}, \ldots, \tau_{N}^{u}\right\}=\Gamma_{T^{\prime}}^{u}
$$

(N.B. The delays produced remain after the removal of $u$ providing us with a 'neural-latching' mechanism.)

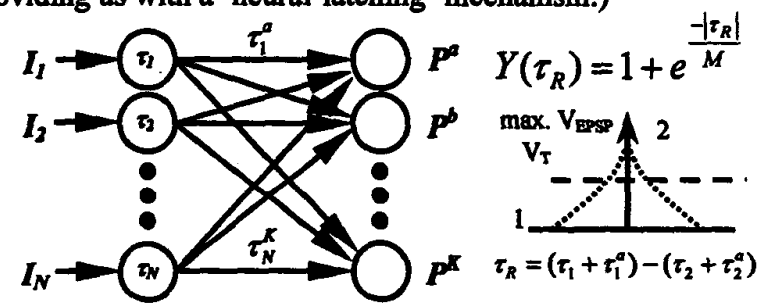

Figure 2 (a): Network architecture

Figure 2(b):Activation function

Each output has excitatory connections from all oscillators (Fig. 2(a)) with delays equal to the relative time differences associated with the stored pattern. A perfect match results in all $N A P S$ coinciding at the output for that input pattern.

\section{RESULTS}

Fig. 2(b) shows how the maximum of the summed EPSPs $Y\left(\tau_{R}\right)$ (of the form $v(t)_{\text {EPSP }}=\exp (-1 / M) \Theta(t)$ where $Q(t)$ is the Heaviside function and $M$ the membrane time constant) generated vary with time difference between $A P S$ for $N=2$ at an output neuron with delays matched for pattern $a$. Tolerance with regard to pattern matching can be varied using a threshold e.g. for $Y\left(\tau_{R}\right)>V_{T}$ the neuron fires signalling a match.

\section{Discussion}

It has been shown that a TNN based on action potential timing using perturbed oscillatory physiologically realistic model neurons is capable of successful pattern recognition. REFERENCES

1 J.J. Hopfield, "Pattern recognition computation using action potential timing for stimulus representation", Nature, Vol-376, pp.33-36, 6 July 1995.

2 A. M. de Paor, C. D. Murray, "Simulation studies in rehabilitation leading to simplification of the Hodgkin-Huxley neuron", Lékar a Technika, vol. 28, pp. $51-60,1997$

3 A.T. Winfree, The Geometry of Biological Time, NY,Springer-Verlag, 1980 